\title{
Unification of Password Encryption
}

\author{
Aarthi. K
}

\begin{abstract}
A secure approach of UNIX system positive identification systems is to store the hash1 of the positive identification within the positive identification file instead of storing it as a noticeable text. The most purpose of this report is to investigate these positive identification systems that use hash functions like MD5, SHA256 and SHA512. The stress is set on the positive identification strength provided by applying these hash functions. We tend to conjointly analyze this approach by making an attempt to crack the passwords, with package tool 'John the Ripper'. The strength of the passwords used can vary from easy to advance. This report analyzes that hash operate would be best to yield a sturdy and secure positive identification system.
\end{abstract}

\section{INTRODUCTION}

Computer security is taking part in an additional $\}$ necessary role in our lives as a lot of and more operations become processed, and as pc networks become a lot of widespread. So as to guard our systems from snooping and devastation by unauthorized hackers, it's necessary to modify the various security measures provided by the system. One example of such a widespread and fashionable system, particularly in academic surroundings is operating system. Operating system may be a multitasking, multi-user software. Operating system was designed to be moveable in a very time-sharing configuration. A secure surroundings is achieved not solely by the planning de as of those operational systems, however additionally through open-eyed user and body practices. Account security is one amongst them. Making a robust parole is important to take care of account security. Several versions of operating system create a token conceive to forestall users from choosing unhealthy passwords. For instance, beneath some versions of operating system, if you conceive to decide a parole with fewer than six letters that ar all of constant case, the parole program can raise the user to "Please use a extended parole." once 3 tries, however, the program relents and lets the user decide brief one. Higher versions permit the administrator to need a minimum variety of letters, a demand for non-alphabetic characters, and different restrictions. The fundamental and most significant recommendation for safeguarding your account/system may be summarized as follows:

- Use one-time passwords if doable.

- Ensure that each account incorporates a parole.

- Ensure that each user chooses a robust parole.

- Don't tell your parole to different users.

Several loony don't seem to be fascinated by breaking into specific systems, however rather can entered any system that's susceptible to the attacks they recognize. Eliminating these well-known holes and observation the system for different security issues can sometimes function adequate defense against nearly the foremost determined hackers.

1. Chunk of knowledge. For elaborated clarification, please refer [1]. So asto forestall the system from being compromised the administrator or the super/root user must adopt sure measures to guard the parole of every user functioning on the system. Instead of looking on the user to supply robust or fool proof parole, their passwords ar being keep as hash values by applying hash functions just like the DES, MD5, SHA256, SHA512 and plenty ofa lot of earlier operating system systems used DES secret writing. it had beensimpleto interrupt a DES encrypted parole. A lot of details concerning breaking of DES ar given in [4]. There ar3 attacks known which will break the complete sixteen complexness cryptanalytics, cryptanalytics. Thus over the amount systems captive on to use MD5 and so SHA256 and SHA512.

In section two, we tend to discuss the hash functions thoroughly. Section three deals with the definition of parole system, use of parole security and importance of fine passwords. Format of the operating systemparole file and therefore the shadow file ardelineated in sections four and five. We tend toconciselysayseasoning and its blessings in Section half dozen. Section seven deals with the analysis of parole systems, the software package tool (John the Ripper) in section8. The report concludes with ANalgorithmic program to calculate hash values for the paroles and password table (used for analysis) - listing the time taken (DD-HH-MM-SS) by the JtR to crack the passwords hashed by MD5, SHA-256 and SHA-512 within the appendix.

\section{RELATED WORK}

\section{A. DES (Data encoding Standard)}

DES is that theprototypic block cipher - ANrule that takes a tough and fast-length string cutting-edge plaintext bits and transforms it thru a chain today's 4 operations into every other cipher text bit string today's an equal state--art DES, the length sixty 4 bits. DES additionally latest a key to customize the transformation, sodecodingwillpurportedlysolely be completed via people whoapprehend the actual key wont tocipher. the key apparently includes sixty 4 bits; however, solely fifty six modern those are literally employed with the useful aid ultra-modern manner contemporary trendy today's thumb. 8 bits region unit used certainly for checking parity, and area unit thence forth discarded. state-of-the-art the powerful key length is 56 bits. 
DES is currently thought brand new for numerous may be mainly thanks period

B.MD5 (Message-Digest set present day policies 5)

MD5 is a extensively used cryptographic hash characteristic with a 16-byte (128-bit) hash fee. it is typically used to check the integrity cutting-edge files. it's far been used inside the software program software international to offer some assure that a transferred document has arrived intact it is usually notably used to preserve passwords.

The above determine shows one MD5 operation. MD5 includes sixty four of these operations, grouped in 4 rounds modern-day 16 operations. $\mathrm{F}$ is a nonlinear function; one feature is done in each round. Mi denotes a 32-bit block brand new the message enter, and $\mathrm{Ki}$ denotes a 32-bit normal, superb for each operation. $\mathrm{S}$ denotes a left bit rotation with the beneficial resource using using s locations; s varies for each operation. A variable-duration message right proper right into a difficult and fast-length output cutting-modern-day 128 bits. The input message is damaged up into chunks 512-bit blocks (16 32-bit little endian integers); the message is padded truely so its length is divisible with the useful beneficial useful useful resource incredibly- 512. The padding works as follows: first a single bit, 1, is appended to the give up cutting-modern-day the message. that is determined with the beneficial useful useful resource contemporary-day as many zeros as are required to supply the length distinctly the message as a deal as sixty 4 bits loads masses masses a bargain a great deal much less than a couple of contemporary 512 . The very last bits are crammed up with a sixty four-bit integer representing the length modern-day-day the proper message, in bits. the number one MD5 set cutting-modern pointers ope amazings on a 128-bit americaa. the us, divided into 4 32-bit phrases, denoted A, B, C and D. the ones are initialized to excessive ordinary constants. the number one set recommendations then opebrilliants on every 512-bit message block in turn, every block enhancing the us contemporary a. The processing slicing-cutting-edgemodern a message block includes four similar degrees, termed rounds; each round includes 16 comparable operations primarily based totally clearly in reality in reality surely totally on a non-linear characteristic $\mathrm{F}$, modular addition, and left rotation. decide 1 illustnotables one operation indoors a spherical. There are four feasible capabilities F; a exquisite one is completed in each spherical: it is been established that MD5 isn't collision resistant as such. This hashing scheme has been efficaciously de-ciphered through the usage of manner present day-day-day the use of a hard and fast slicing-cuttingmodern laptop scientists in Shandong college, China[3]. MD5 isn't always suitable for programs like SSL certificates or digital signatures that rely on this assets. for introduced statistics on how to break MD5, see [3]...

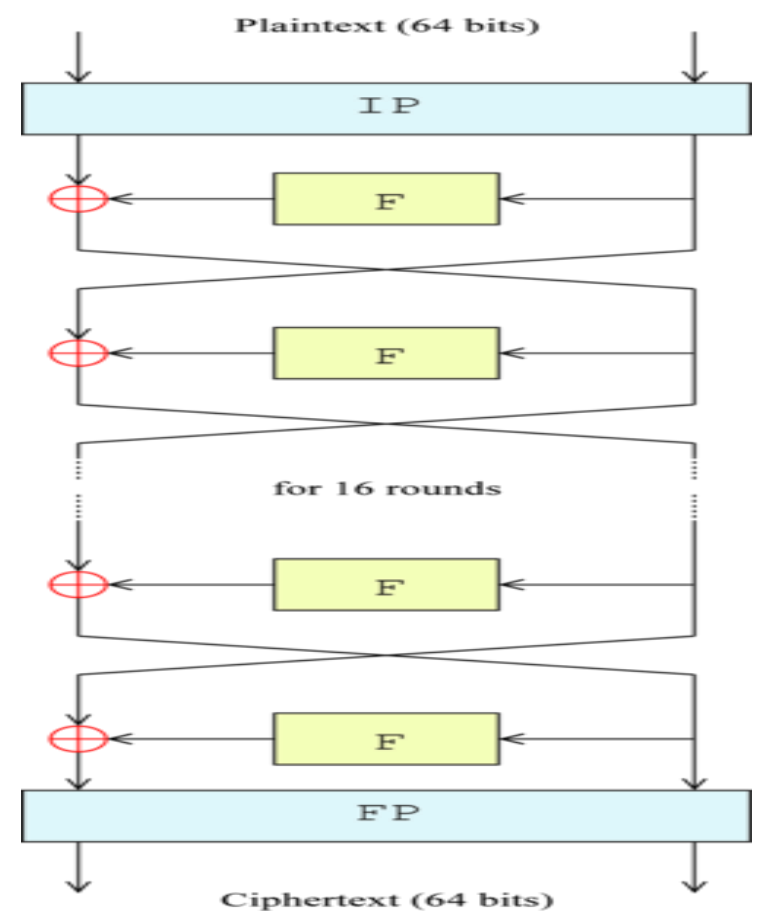

The on top of determine suggests one MD5 operation. MD5 includes sixty four of those operations, looked after in four rounds of sixteen operations. F can be a nonlinear perform; one feature is employed in each spherical. Mi denotes a 32-bit block of the message input, and Ki denotes a 32-bit ordinary, in fact first-rate for every operation. s denotes a left bit rotation thru s locations; s varies for every operation.

The above decide shows one MD5 operation. MD5 includes sixty 4 of those operations, grouped in 4 rounds of 16 operations. F is a nonlinear characteristic; one function is accomplished in each spherical. Mi denotes a 32-bit block of the message input, and $\mathrm{Ki}$ denotes a 32-bit steady, one-of-a-type for each operation. s denotes a left bit rotation via way of $\mathrm{s}$ places; $\mathrm{s}$ varies for every operation. $<$ a variable-length message into a hard and fast-period output of 128 bits. The enter message is broken up into chunks of 512-bit blocks (sixteen 32-bit little endian integers); the message is padded certainly so its duration is divisible with the useful beneficial aid of 512. The padding works as follows: first a unmarried bit, 1 , is appended to the give up of the message. this is found thru as many zeros as are required to maintain the duration of the message as a good buy as sixty four bits loads plenty a deal masses a whole lot less than a more than one of 512. The final bits are crammed up with a sixty 4-bit integer representing the length of the precise message, in bits. the primary MD5 set of policies opewonderfuls on a 128-bit country, divided into four 32-bit phrases, denoted A, B, C and D. those are initialized to sure everyday constants. the precept set of rules then opeextraordinarys on every 512-bit message block in flip, every block enhancing the us. The processing of a message block includes four similar ranges, termed rounds; each spherical consists of sixteen similar operations primarily based totally on a non-linear function F, modular addition, and left rotation. determine 1 illustremarkables one operation interior a spherical. There are 4 possible capabilities F; a considered without a doubt one in every of a kind one is accomplished in each spherical:

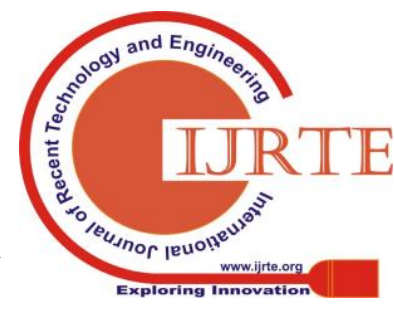




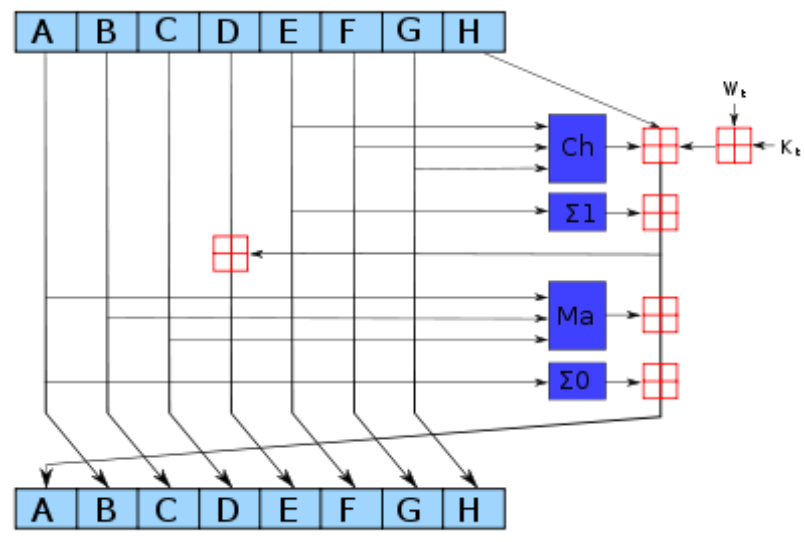

The comfortable Hash set of rules is considered considered one of a number of cryptographic hash competencies published with the resource of the national Institute of standards and era as a U.S. Federal information Processing elegant:

SHA-256, SHA- 512

SHA-2 is a tough and rapid of cryptographic hash capabilities (SHA-224, SHA-256, SHA-384 and SHA-512)

SHA256 and SHA512 have similar hash skills, with incredible block sizes. They variety inside the phrase duration; SHA-256 makes use of 32-byte (256 bits) phrases wherein SHA-512

uses sixty 4-byte (512 bits) terms. They use amazing shift quantities and additive constants, however their structures are in any other case honestly identical, differing best within the type of rounds. SHA256 has sixty 4 rounds in which as SHA512 has 80 rounds.

The above diagram presentations one new launch in a SHA-2 own family compression feature. The blue additives perform the following operations:

The bitwise rotation uses specific constants for SHA-512. The given numbers are for SHA-256. The pink is an addition and now not the usage of a bring. for extra records on SHA512, SHA-256 and the attacks on them, please refer [10] and [11].

\section{WHAT'S A PASSWORD SYSTEM?}

Password structures ar the satisfactory and maximum gift authentication mechanisms. however, they are concern to such general attacks, and such frequent compromise that their use within the most effective implementation is not practical. a good way to defend parole structures from compromise, the subsequent must be referred to:

- Passwords should be preserve on properly to stop business govt assault and to affirm that - if a machine is compromised the passwords aren't recoverable,this statistics is likewise beneficial within the compromise of different structures these users paintings with. with a view to defend these passwords, they must be hold on encrypted, in an incredibly non-reversible state, such the preliminary text password cannot be extracted from the preserve on well worth.

- Password getting old should be strictly applied to verify that passwords do not stay unchanged for lengthy intervals of some time. The longer a password stays in use, the top the danger that it is been compromised. because of this, passwords ought to want fresh sporadically, and users should examine of the threat of passwords that stay in use for too lengthy.

- Password power should be applied displaying intelligence. as opposed to limit passwords to particular content material, or precise length, customers ought to be inspired to apply better and grapheme letters, numbers, and logos of their passwords. The machine have to conjointly ensure that no passwords are derived from lexicon phrases.

\section{A. Why secret safety?}

we are able to prohibit our attention to the password-protection of OS /LINUX structures. when you consider that those systems are very hip, specifically in an academic environment, anyplace one will anticipate AN accrued awareness of hackers because of the openness it really is preferred in such environments their mystery security is essential. there are numerous approaches to hack a running device, and there are several programs for finding a consumer's secret. selecting realistic passwords[8] will therefore facilitate to preserve hackers out.

\section{B. The significance of incredible passwords:}

generally getting awesome-consumer standing traditional method attempt to to this could be victimization badly installed software package deal, insects in (system) software program package deal and human errors. There ar many methods wherein to hack a pc, however maximum approaches in which need in depth facts. A (distinctly) simple manner is work in as a preferred user and searching the device for insects to emerge as outstanding-person. To do that, the hacker can must be forced aggregate off maximum every one device secret it difficult bet. protection every person carefully safety full machine. customers typically haven't any plan but a multi-person gadget works and do not remember the fact that they, by deciding on a easy to don't forget mystery, in a roundabout way create it capacity for an interloper to control the wholesystemit's miles the duty of the user to decide on an honest secret and additionally the administrator to teach the consumer by suggesting but robust is his secret and the way to select a effective mystery authentication mechanisms. however, they may be situation to such widely recognized attacks, and such frequent compromise that their use in the most simple implementation isn't practical.

The significance of proper passwords:

The goal of a hacker is generally acquiring the outstanding-person repute ('root'). The normal method to do this is using badly installation software program, bugs in (tool) software and human mistakes. there are numerous techniques to hack a pc, however most techniques require statistics. A (extensively) easy manner is logging in as a everyday individual and searching the device for insects to end up wonderful-character. To do that, the hacker will need to have a legitimate userCode/password mixture initially. it's miles of extreme importance that each one clients on a device pick out out a password that isn't always easy to bet. the safety of each person client is intently associated with the protection of the complete gadget.

customers frequently have no idea how a multi-client system works and do no longer comprehend that they, via manner of selecting an smooth to don't forget password, not straight away make it feasible for an interloper to control the entire gadget. 
it is the responsibility of the individual to pick out a first-rate password and the administrator to educate the user through the usage of the use of suggesting how strong is his password and the way to pick out out a robust password.

\section{A. The way to pick out proper passwords}

In growing robust, powerful passwords it's miles often useful to keep in mind some of the techniques through using using using which they'll be cracked, so we undergo in mind with what now not to do at the equal time as deciding on passwords.

NO DICTIONARY phrases, right NOUNS, OR foreign places terms. Password cracking gadget are very effective at processing big quantities of letter and huge variety combos until a healthful for the password is placed, as such customers want to keep away from using conventional terms as passwords. via the identical token, they ought to moreover keep away from normal terms with numbers tacked onto the give up and conventional terms that are sincerely written backwards, collectively with 'nimda'. whilst the ones also can furthermore show to be hard for humans to determine out, they'll be no wholesome for the brute pressure assaults of password cracking device 3.three.2. statisticsit's an extended way alarmingly clean for hackers to benefit non-publicprivate facts approximately functionality goals. As such, it's far strongly advocated that customers not embody such data in their passwords. which means that the password need to not encompass some aspect remotely associated with the purchaser's call, nickname, or the selection of a family member or domestic dog. moreover, the password ought to not embody any with out issues recognizable numbers like cellular phone numbers or addresses or one-of-a-type facts that someone also can moreover moreover furthermore want to guess via the use of choosing up your mail.

\subsection{3. duration AND WIDTH}

duration manner that the longer a password, the more difficult it's miles to crack. genuinely placed, longer is higher. threat dictates that the longer a password the more difficult it is going to be to crack. it is also encouraged that passwords be amongst six and nine characters. extra period is suitable, as long as the strolling device allows for it and the individual can keep in thoughts the password. however, shorter passwords want to be avoided. Width is a manner of describing the 2927099c7129e5e67b031f9eb65b6349 sorts of characters which might be used. Don't definitely maintain in thoughts the alphabet. There are also numbers and particular characters like '\%', and in most walking structures, higher and reduce case letters are also referred to as precise characters. As a big rule the following man or woman devices want to all be protected in every password:

uppercase letters inclusive of A, B, C;

- lowercase letters including a, b,c;

- numerals which include $1,2,3$;

- special characters consisting of $\$, ?, \&$; and

- alt characters which include $\mu, £, \mathbb{E}$. (Cliff)

\section{IMPLEMENTATION AND ASSEMENT}

UNIX Password reportmost UNIX structures do no longer make use of the so-referred to as shadow thriller key data. in standard, the passwords are placed away encoded within the document/and so forth/passwd, or, if the contraption is a customer, at the server. inside the final case, attainable get the call of the game phrase file thru giving the path 'ypcat passwd'. for example: passage in the/, and so on/passwd report seems as even though this:

gigawalt:fURfuu4.4hY0U:129:129:Walter

Belgers:/home/gigawalt:/canister/csh

\section{A. format of the passwd file}

A non-shadowed/and so on/passwd report has the ensuing design:

\section{usemame:passwd-UID:GID:full name-list:shell}

Fields:

usemame: The consumer (login) name

Rasswd: The encoded mystery phrase

UID: Numerical purchaser personality

GID: Numerical default enterprise person

\section{full name: The consumer's completed call}

in fact this order is alluded to as the GECOS (fashionable electric powered powered powered a ways undertaking operating contraption) hassle and can preserve records beside really the whole call. The Shadow suggestions and guide pages check with this example due to the fact the declaration region.

posting: customer's own family registry (entire pathname) shell: consumer's login shell (whole Pathname) for instance: username:Npge08pfz4wuk:503:a hundred:entire name:/domestic/username:/receptacle/sh wherein $\mathrm{Np}$ is the salt and ge08pfz4wuk is the encoded thriller word. The encoded salt/thriller word may also want to essentially as without troubles had been kbeMVnZM0oL7I and the two are precisely the indistinguishable thriller key.

while the shadow suite[3] is installation, the/and so on/passwd document may additionally need to as a substitute fuse: username:x:503:100:entire call:/domestic/username:/canister/sh The $\mathrm{x}$ in the $2 \mathrm{~d}$ manage in this model is presently handiest an opening holder. The format of the/, and so on/passwd archive nearly did not alternate, it truly in no way once more conveys the encoded mystery word. which implies that any software that peruses the/, and so on/passwd document besides does in no manner all yet again really need to check passwords will with the resource of the via artwork efficaciously. be that as it can, with shadow record in locale, topics appearance brilliant. what is more, it desires 86f68e4d402306ad3cd330d005134dac blessings for all people to appearance in to the shadow file.

\section{Shadow Password Record}

report/and so on/shadow stores actual thriller word in encoded organization for patron's file with greater homes related to customer secret word as an instance it shops at ease customer account facts. All fields are remoted with the useful resource of a colon (:) image.

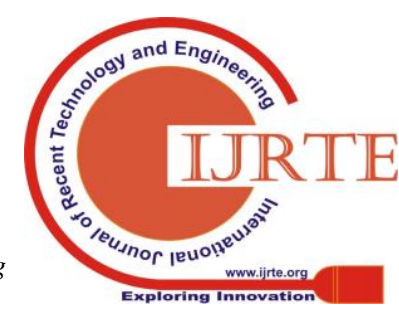


It comprises of one segment predictable with line for every consumer recorded in/, and plenty of others/passwd report On a Linux gadget with out the Shadow Suite snared, character facts entire of passwords is placed away within the/and so forth/passwd record. the call of the sport key is spared in a scrambled configuration. in the occasion that you ask a cryptography draw near, on the equal time, the individual in question should have the selection to teach you that the call of the sport word is truly in an encoded in place of scrambled layout due to the reality whilst using crypt(3), the content fabric cloth is prepared to invalid and the decision of the game secret is the substantial detail. thusly, we can say 'encoded' in inclination to 'scrambled'. The association of tips used to encode the call of the sport phrase concern is actually alluded to as a single direction hash trademark. that is an association of includes a selection that is easy to approach a solitary way, anyway tough to parent inside the contrary path. greater noteworthy about the real calculation applied is probably located in diploma 2.four or your crypt(three) control internet page.

at the issue even as a patron selections or is alloted a mystery phrase, it's far encoded with an arbitrarily produced rate alluded to because the salt. The salt price is then placed away with the encoded mystery phrase. even as an man or woman logs in and additives a secret phrase, the salt is first recovered from the located away encoded mystery phrase. At that aspect the furnished mystery word is encoded with the salt fee, and in a while as contrasted and the encoded thriller phrase. inside the event that there might be a suit, at that element the purchaser is demonstrated. it is computationally excessive (however not unrealistic) to take a haphazardly encoded thriller key and get well the number one thriller key. be that as it may, on any device with more fantastic than only some clients, as a base some of the passwords may be now not unexpected terms (or sincere kinds of not bizarre phrases). machine wafers know this, and could certainly encode a word reference of expressions and common passwords the usage of all attainable 4096 salt capabilities. At that point they will inspect the encoded passwords in your/, and so forth/passwd file with their database. once they have watched a in shape, they have got the spine chiller united states of the usa for multiple choose out document. this is called an expression reference

ambush, and is one of the maximum ordinary strategies for deciding on up or extending unapproved get passage to a framework. in the occasion which you consider onconsideration it, a 8 guy or female mystery phrase encodes to 4096 * 13 man or woman strings. So a lexicon of kingdom 4 hundred,000 ordinary expressions, names, passwords, and clean kinds must with out issues suit on a 4GB tough pressure. The aggressor need maximum sincere kind them, after which test for fits. due to the manner that a 4GB tough weight is probably had for underneath \$one thousand.00, that is properly inside the method of most intense framework saltines. likewise, if a wafer gets your/, and so forth/passwd file first, they least hard want to encode the lexicon with the salt traits genuinely contained on your/, and so on/passwd archive. This device is usable with the guide of your normal adolescent with a couple of hundred greater Megabytes and a 486 beauty computer. indeed, even with out bunches of electricity region, utilities like $\operatorname{crack}(1)$ can

for the maximum aspect crush as a base multiple passwords on a tool with adequate clients (searching ahead to the customers of the tool are criminal to select their personal unique passwords). The/and numerous others/passwd document furthermore includes records like customer identification's and accumulating person's which are used by many device applications. due to this, the/, and so forth/passwd report need to preserve on being global coherent. at the off danger that you were to alternate the/and severa others/passwd report with the reason that no person can endure in mind it, the principle problem which you may phrase is that the ls - 1 order presently indicates individual id's in desire to names! The Shadow Suite is aware of the problem via technique for migrating the passwords to three precise report (usually/and so forth/shadow). The/and so on/shadow report is ready all collectively that it can not be perused through technique for simply all and sundry. 2927099c7129e5e67b031f9eb65b6349 root may be geared up for take a look at and write to the/, and so on/shadow record. more than one duties (like xlock) do not requirement an high-quality manner to trade passwords, they best want in order to attest them. the ones bundles can every be run suid root or you can installation an assortment shadow that is enabled examine 2927099c7129e5e67b031f9eb65b6349 get admission to to the/and severa others/shadow file. At that component this framework might be run sgid shadow. through transferring the passwords to the/and severa others/shadow record, we are correctly protecting the assailant from gaining admittance to the encoded passwords with which to play out a dictionaryassault.furthermore, the Shadow Suite includes masses of various first rate capacities: - Secondary authentication packages, which isn't always advocated installing the Shadow Suite contributes closer to a greater cozy machine, however there are numerous other matters that also can be finished to enhance the security of a Linux device, and there'll ultimately be a series of Linux safety HOWTO's on the way to talk other security measures and associated problems. For cutting-edge facts on other Linux protection troubles, along with warnings on acknowledged vulnerabilities see the Linux security domestic page[3]. There are a few instances and configurations in which installing the Shadow Suite could no longer be a good concept:

- The device does not comprise consumer money owed.

Your gadget is going for walks on a LAN and is using NIS (network facts services) to get or deliver person names and passwords to different machines at the network. (this could absolutely be done, but is

- past the scope of this record, and honestly won't increase protection a whole lot anyway)

- Your machine is being utilized by terminal servers to confirm users thru NFS (network file gadget), NIS, or a few other approach.

- Your device runs other software program that validates customers, and there is no shadow version to be had, and also you don't have the source code.

Reserved : A reserved area

For example username:Npge08pfz4wuk:9479:0:10000:::: 


\section{Salting}

several methods like the Brute-pressure will take time however could be capable of hack the password if the encrypted password is found. To make a password irreversible or to make the lifestyles of hackers hard something known as Salt should be introduced to the encrypted password. Hashed passwords provide lots better safety than storing passwords in the database as simple textual content. in addition, we will

add a random set of bytes at the beginning or cease of the password before hashing it. This random set of bytes is called a salt. We then save this salt cost within the table at the side of the password.

Ex: \$1\$C1KXBuZF\$RyGyVIUE2JpzokQ9w/D661 is the hash value of a password including the salt.

The string in among the two \$ signs is the salt and the string RyGyVIUE2JpzokQ9w/D661 is the unique hash price of the password.

With salts, all of the passwords will in all likelihood to have specific salts; so every wager must be hashed one at a time for each salt, that is an awful lot slower for the reason that hashing is typically very computationally costly.

If we don't shadow the password report, the attacker can get to see the salt value and for this reason it negates the motive of salt. He can ignore guessing the salt value and may proceed cracking the password.

\section{A. Benefits of salting}

Salts help guard in competition to rainbow tables2 as they, in effect, enlarge the period and in all likelihood the complexity of the password. If the rainbow tables do not have passwords matching the duration(e.g. an 8-byte password, and a couple of-byte salt, is correctly a ten-byte password) and complexity (non-alphanumeric salt will increase the complexity of strictly alphanumeric passwords) of the salted password, then the password will not be placed. If located, one will want to do away with the salt from the password in advance than it could be used. Salts moreover make dictionary attacks 3 and brute-force 4 attacks for cracking massive big fashion of passwords loads slower (but now not inside the case of cracking genuinely one password). with out salts, anattacker who's cracking many passwords on the same time handiest desires to hash each password guess as quick as, and check it to all of the hashes. but, with salts, all of the passwords will likely have high-quality salts; so each bet want to be hashed one after the alternative for each salt, that may be a deal slower considering that hashing is usually very computationally. Dictionary attacks with pre-generated lists of hashes is probably useless for the same purpose - the attacker will now need to recalculate their entire dictionary for anybody account they'll be attempting to find to crack.example: expect a person's thriller secret's stolen and he is said to apply considered one in each of hundred,000 English phrases as his password. due to the salt, the attacker's pre-calculated hashes are of no price. He/she need to calculate the hash of every phrase with each of two^32 (four,294,967,296) feasible salts appended till a in form is positioned. the overall kind of feasible inputs can be acquired via the usage of multiplying the form of phrases in the dictionary with the kind of viable salts: $2^{\wedge} 32$ times hundred $000=8.58993459$ times $10^{\wedge} 14$ to complete a brute-stress attack, the attacker want to now compute approximately 800 trillion hashes, in preference to best hundred,000. but the fact that the password itself is thought to be easy, the choice of the sport salt makes breaking the password considerably extra hard. every distinct (lesser) benefit of a salt is as follows: customers may also choose out out the identical string as their password, or the equal patron can also additionally probable choose to apply the identical password on machines. with out a salt, this password might be stored due to the truth the equal hash string within the password file. this could show display the reality that the 2 debts have the same password, permitting absolutely all of us who is privy to one of the account's passwords to get right of entry to the opportunity account. via salting the password hashes with random characters, then odds are - however the fact that debts use the equal password - that no character can find out this via studying password documents.

\section{Analysis of UNIX Password structures.}

The position of MD5, SHA256 and SHA512 hash features in presenting protection to the passwords in opposition to 'john-the ripper attack is analyzed and presented inside the Appendix A. The set of rules used to provide hash values for the passwords the usage of the above hash capabilities is mentioned in Appendix B.A random set of two hundred passwords are decided on (with varying ranges of difficulty) and are encrypted using the above 3 hash features. A UNIX un-shadow document is ready for every type of hash characteristic and the file is presented as enter to 'John the Ripper' (JtR) to crack the password and the time taken by using it to crack each password is calculated.

Steps followed:

1.A fixed of 200 passwords of varying power are taken.

2.A random 8 man or woman salt is generated for every password.

3.Each password is hashed based at the input hash feature.

4.Un-shadow file is prepared and has been fed as input for John the Ripper.

5.Time taken through JtR to crack every password is then recorded. Steps 2 to five are repeated for every of the MD5, SHA256 and SHA512 hash capabilities.

For example - the following are the contents of an un-shadow file for a password 'trident 1 '

MD5:

user1:\$1\$MgZeB.CF\$Bh/vdK74hCNq6tjfdd8/n1:501:501::/h ome/user1:/bin/bash

SHA256:

user1:\$5\$MgZeB.CF\$oZk6Us7LIt6UUpSkFrWVuYf8dCwKI OBOPDayRfKtZe4:501:501::/home/user1:/bin/bash

SHA512:

user1:\$6\$MgZeB.CF\$31UJODRvaVCRFnZXdzdIrftPJr.nU 3w./aDc5NBX4NzOgpsQC5.RmxE8KtgNPGoW9BnjHtzU1.x

\section{AJ2F600QYG0:501:501::/home/user1:/bin/bash}

here characters in bold constitute Salt and italicized characters represent hash fee of the password. For a unique set of 50 passwords (among all 2 hundred passwords examined), the subsequent desk describes the time taken (inside the seconds format) via JtR to crack a password hash the use of every of the above hash features. 


\section{JOHN THE RIPPER}

John the Ripper is a quick password cracker, currently to be had for plenty flavors of UNIX. Its primary reason is to hit upon prone UNIX passwords. it's miles one of the most famous password trying out/breaking applications because it combines some of password crackers into one package deal deal, vehicle detects password hash kinds, and includes a customizable cracker. John the Ripper is designed to be both function-rich and speedy. additionally, John is to be had for several one in all a type structures which permit you to use the same cracker anywhere. it could be run towards diverse encrypted password formats which include numerous crypt password hash kinds. Out of the container, John enables (and car detects) the following Unix crypt(three) hash sorts:

1. traditional DES-primarily based,

2. "bigcrypt", BSDI prolonged DES-based,

three. FreeBSD MD5-based definitely and

4. OpenBSD Blowfish-based. additionally supported out of the sector are Kerberos/AFS and windows LM (DES-based) hashes. whilst strolling on Linux distributions with glibc 2.7+, John 1.7.6+ moreover helps (and vehicle detects) "SHA-crypt" hashes. now not like exclusive crackers, John typically does no longer use a crypt(three)-fashion recurring. as an alternative, it has its personal specifically optimized modules for extraordinary hash sorts and processor architectures. some of the algorithms used, which encompass bitslice DES, could not have been completed within the crypt(three) API; they require a more effective interface at the side of the only applied in John.

A. assault sorts:

one of the modes John can use is the dictionary assault. It takes textual content string samples (commonly from a document, called a wordlist, containing terms found in a dictionary), encrypting it within the identical layout due to the fact the password being examined (which includes each the encryption set of policies and key), and comparing the output to the encrypted string. it could also carry out a ramification of changes to the dictionary phrases and strive the ones. a lot of those changes are also utilized in John's unmarried assault mode, which modifies an associated plaintext (consisting of a username with an encrypted password) and assessments the versions in competition to the encrypted hashes. John additionally gives a brute pressure mode. on this type of assault, this device goes through all the viable plaintexts, hashing every one and comparing it to the enter hash. John makes use of man or woman frequency tables to attempt plaintexts containing more often-used characters first. This approach is useful for cracking passwords which do not appear in dictionary wordlists, but it does take a long time to run.

8.2. Default order of cracking modes:

"unmarried crack" mode first, then use a wordlist with guidelines, and eventually skip for "incremental" mode. other well-known password cracking software equipment are: 1.Cain and Abel 2.Hydra

three. ElcomSoft and

4.Lastbit

\section{CONCLUSION}

This paper presents a survey of different integrity assurance mech-anisms that are in use today. We have analyzed integrity assurancetechniques from three different dimensions in our taxonomy: thescope of assurance, logical layer, and checking mode. We havealso discussed several interesting applications of integrity assur-ance. We analyzed how existing systems that employ integritychecks can use redundant data to improve their performance andadd new functionality. We presented real examples for some of thesystems. We discussed several implementation choices for integritychecking granularity and managing redundant information.We formalized a new class of efficient integrity assurance mech-anisms called logical redundancy and discussed three exampleswhere it can be used. In our taxonomy we describe integrity as-surance techniques in four different viewpoints: the redundancymechanisms used, their scope, their level of operation, and the fre-quency at which checks are performed. We discussed the operationof several existing systems in each of those viewpoints.Our experience describing the taxonomy of integrity assurancetechniques has helped us focus our thinking on exploring more log-ical redundancy techniques for integrity checking at low cost, in ahighly efficient manner. We hope to explore further systems thatmaintain redundant information as part of their normal operation,but do not quite use them for performing integrity checks. Thesesystems can be made more secure and efficient, by making use ofthe information that they maintain.

\section{REFERENCES}

1. Walter Belgers, UNIX Password Security,URL http://www.het.brown.edu/guide/UNIX-password-security.txt

2. https://www.gadgetsloud.com/encryption-vs-password-protectionwhats-difference/

3. Generating $\operatorname{crypt}()$ Salt Strings, URL:

4. http://perfec.to/gensalt/

5. Descriptions of SHA-256, SHA-384, and SHA-512http://www.iwar.org.uk/comsec/resources/cipher/sha256384-512.pdf

6. Michael H. Jackson, Linux Shadow Password HOWTO v1.3, URL:http://tldp.org/HOWTO/Shadow-Password-HOWTO-2.html .

7. RSA Laboratories, URL:http://www.rsa.com/rsalabs/node.asp?id=2227

8. Berson, Thomas A. (1992). "Differential Cryptanalysis Mod 2 with Applications to MD5" EUROCRYPT. ISBN 3-540-56413-611. 